\title{
Disease modifying agents of myeloproliferative neoplasms: a review
}

\author{
Sung-Eun Lee \\ Department of Hematology, Seoul St. Mary's Hospital, College of Medicine, The Catholic University of Korea, Seoul, Korea
}

p-ISSN 2287-979X / e-ISSN 2288-0011

https://doi.org/10.5045/br.2021.2020325

Blood Res 2021;56:S26-S33.

Received on December 18, 2020

Revised on March 25, 2021

Accepted on March 30, 2021

\begin{abstract}
The identification of driver mutations in Janus kinase $(J A K) 2$, calreticulin $(C A L R)$, and myeloproliferative leukemia $(M P L)$ has contributed to a better understanding of disease pathogenesis by highlighting the importance of JAK signal transducer and activator of transcription (STAT) signaling in classical myeloproliferative neoplasms (MPNs). This has led to the therapeutic use of novel targeted treatments, such as JAK2 inhibitors. More recently, with the development of next-generation sequencing, additional somatic mutations, which are not restricted to MPNs, have been elucidated. Treatment decisions for MPN patients are influenced by the MPN subtype, symptom burden, and risk classification. Although prevention of vascular events is the main objective of therapy for essential thrombocythemia (ET) and polycythemia vera (PV) patients, disease-modifying drugs are needed to eradicate clonal hematopoiesis and prevent progression to more aggressive myeloid neoplasms. JAK inhibitors are a valuable therapeutic strategy for patients with myelofibrosis (MF) who have splenomegaly and/or disease-related symptoms, but intolerance, refractory, resistance, and disease progression still present challenges. Currently, allogeneic stem cell transplantation remains the only curative treatment for MF, but it is typically limited by age-related comorbidities and high treatment-related mortality. Therefore, a better understanding of the molecular pathogenesis and potential new therapies with the aim of modifying the natural history of the disease is important. In this article, I review the current understanding of the molecular basis of MPNs and clinical studies on potential disease-modifying agents.
\end{abstract}

Key Words Myeloproliferative neoplasms, Polycythemia vera, Essential thrombocythemia, Myelofibrosis

\section{INTRODUCTION}

Classical myeloproliferative neoplasms (MPNs), also called Philadelphia-negative MPNs, are clonal hematopoietic disorders characterized by the excessive production of terminally differentiated blood cells [1]. Classical MPNs include three main diseases: PV, ET, and MF, which have frequent disease-related complications such as venous and arterial thrombosis, hemorrhages, and transformation to acute myeloid leukemia [2]. The identification of driver mutations in JAK2, CALR, and MPL has contributed to a better understanding of disease pathogenesis, implicating near-universal upregulation of JAK-STAT signaling [2, 3], and has led to the development and therapeutic use of novel targeted treatments, such as JAK2 inhibitors [4-6]. More recently, according to the development of next-generation sequencing, additional somatic mutations have been identified that are not restricted to MPNs and occur in other myeloid malignancies, including acute myeloid leukemia (AML) [7, 8], myelodysplastic syndrome (MDS) [9, 10], or in some elderly patients without overt myeloid malignancies [11-13]. Moreover, aging, the bone marrow microenvironment, and other genetic factors such as germline predisposition, order of mutation acquisition, and variant allele frequency have been demonstrated as key factors influencing clonal outgrowth [14].

Treatment decisions for MPN patients are influenced by the MPN subtype, symptom burden, and risk classification. Even though the main objective of ET and PV therapy is to prevent vascular events, there is a need for disease-modifying drugs that can eradicate clonal hematopoiesis and prevent progression to more aggressive myeloid neoplasms [15-17]. JAK inhibitors are a valuable therapeutic strategy for patients with MF who have splenomegaly and/or disease-related symptoms $[4,5,18]$. However, allogeneic stem cell transplantation remains the only curative treatment for MF, but 
it is typically limited by age-related comorbidities and high treatment-related mortality $[19,20]$. Therefore, a better understanding of the molecular pathogenesis and potential new therapies to modify the natural course of the disease is important.

Here, the present paper reviews the current understanding of the molecular basis of MPNs and potential disease-modifying therapies.

\section{MOLECULAR LANDSCAPE OF MPNS}

\section{Activation of JAK-STAT signaling}

Constitutive activation of the JAK-STAT pathway is a hallmark of classical MPNs [2, 3]. Documented drivers in classical MPNs include $J A K 2 \mathrm{~V} 617 \mathrm{~F}$ and exon 12 mutations [21-24], mutations in MPL [25], and mutations in exon 9 of CALR [26, 27]. The JAK2V617F mutation is the result of a guanine to thymine somatic mutation at nucleotide 1849 in exon 14 of the $J A K 2$ gene, which leads to a single amino acid substitution from valine to phenylalanine at codon 617 [2]. This mutation is found in 95\% of PV and $50-60 \%$ of ET and primary myelofibrosis (PMF) [21-24]. JAK2 exon 12 mutations have also been found in $1 \%$ to $2 \%$ of PV patients, most of whom have JAK2V617F negative PV [28].

MPL is a cell surface receptor for thrombopoietin (TPO) [29]. MPL mutations at tryptophan W515 located at the boundary of the transmembrane and cytosolic domains of MPL are present in 3\% of ET and 5\% of PMF, with the most frequent mutations being W515L and W515K [30]. Several other substitutions, such as W515R, W515A, and W515G have also been reported [31].

CALR is a protein that resides in the lumen of the endoplasmic reticulum (ER), where it functions as a molecular chaperone for many glycoproteins, assisting in their folding and contributing to calcium homeostasis. The two most frequent mutations are type 1 and type 2 mutations [32, 33]. The type 1 mutation is a 52 bp deletion (c.1092_1143del, p.L367f*46), whereas the type 2 mutation is a 5 bp insertion (c.1154 1155insTTGTC, p.K385fs*47). The distribution of these CALR mutation types varies depending on the MPN subtype; in $\mathrm{PMF}$, the type 1 mutation is more prevalent than type 2 (70\% vs. $13 \%$, respectively), but in ET, type 1 and type 2 mutations are more evenly distributed $(51 \%$ vs. $39 \%$, respectively) [34].

\section{Other somatic mutations}

The recent development of next-generation sequencing has allowed the identification of several mutations in patients with a variety of myeloid neoplasms, including MPNs [2, 35-45]. The most commonly affected genes are those important in epigenetic regulation, messenger RNA splicing, transcriptional mechanisms, and signal transduction. Although reported somatic mutations lack specificity because they can be found in a broad range of myeloid neoplasms, there is evidence to suggest that the identification of certain non-driver mutations in MPN patients is associated with a greater risk of disease progression or shortened survival [19, 46-48].

Mutations in epigenetic regulators: Mutations targeting DNA methylation regulators (TET2, $D N M T 3 A$, and $I D H 1 / 2)$ were identified across MPN subtypes. All TET2 mutations in MPN are loss-of-function point mutations or deletions and are present in approximately $10 \%$ to $20 \%$ of MPN subtypes [49]. $D N M T 3 A$ mutations are less frequent than TET2 mutations in MPNs (5-10\%) [42]. These two types of mutations increase the self-renewal of $J A K 2 \mathrm{~V} 617 \mathrm{~F}$ hematopoietic stem cells (HSCs) and play an important role in disease initiation [50]. They also induce disease progression when they occur as secondary mutations, but their role in myelofibrosis and leukemic transformation remains elusive [50]. Interestingly, IDH1/2 mutations were mutually exclusive with mutations in TET2, and TET2 loss-of-function mutations were associated with similar epigenetic defects as $I D H 1 / 2$ mutants [51].

Mutations in EZH2 occur in $13 \%$ of patients with MF [37]. EZH2 encodes a histone H3 lysine 27 (H3K27) methyltransferase and is the catalytic subunit of the polycomb repressive complex (PRC2), which is required for maintenance of HSCs [52]. Mutations in ASXL1 occur in approximately $25 \%$ of PMF [2], the second most common epigenetic regulator mutation in MPN after TET2 [3, 38]. Similar to EZH2, the loss of ASXL1 impairs PRC2-mediated suppression of leukemia oncogenes in hematopoietic progenitors [53]. As discussed above, the EZH2 and ASXL1 abnormalities result in inhibition of the PRC2 complex, which reduces H3K27 methylation and, in turn, promotes H3K27 acetylation, ultimately exerting a gain-of-function effect through the recruitment of bromodomain and extra-terminal (BET) family proteins [54]. In a JAK2(V617F)/Ezh2-null mouse model, a bromodomain inhibitor significantly attenuated H3K27 acetylation levels at the promoter regions of PRC2 targets and downregulated their expression, leading to the abrogation of MF-initiating cells [55]. Moreover, Kleppe et al. [56] showed that BET inhibitors reduce NF- $\kappa \mathrm{B}$-induced inflammation and bone marrow fibrosis in MPN models, and combination treatment with BET and JAK inhibitors showed improved efficacy.

Mutations in splicing machinery: Mutations in components of the RNA spliceosome machinery, including SF3B1, SRSF2, $U 2 A F 1$, and $Z R S R 2$, are involved in MPN patients, especially ET and MF, as well as other myeloid malignancies [43, 57]. SRSF2 encodes a protein that is a member of the serine/arginine-rich splicing factor family; it binds to exonic splicing enhancer (ESE) sequences in pre-messenger RNA (premRNA). SRSF2 mutations alter SRSF2's normal sequencespecific RNA binding activity, thereby altering the recognition of specific exonic splicing enhancer motifs to drive recurrent mis-splicing of key hematopoietic regulators [58]. In particular, mutations in the spliceosome components U2AF1 and SRSF2 are known to lead to mis-splicing and nonsense-mediated decay of EZH2 $[58,59]$ and are associated with MPNs with poor prognoses [19].

Mutations in transcription factors and signal transduction genes: Sequencing a number of genes implicated in myeloid 
malignancies in samples from patients with post-MPN AML versus chronic MPN phase revealed somatic mutations targeting TP53 tumor-suppressing function in $20 \%$ to $30 \%$ of patients with leukemic transformation, but are uncommon in the chronic phase of MPN [44, 60]. RUNX1 encodes a transcription factor that binds to enhancers and promoters to regulate normal hematopoiesis [61]. Various RUNX1 mutations and chromosomal aberrations are found in $10 \%$ of MPNs to secondary leukemia transitions [45, 62].

Signal transduction gene mutations, such as $N R A S, S H 2 B 3$, $C B L, N F 1$, and FLT3, are associated with an increased risk of leukemic transformation and typically occur more frequently in the blast phase than in the chronic phase of MPN [2, 60, 62].

\section{Molecular-based therapy in ET and PV}

Although the efficacy of interferon- $\alpha$ (IFN- $\alpha$ ) has shown efficacy in the treatment of ET and PV for more than 20 years and has provided an alternative option to chemotherapeutic agents, toxicity and the need for frequent parenteral application of conventional formulations led to a high proportion of patients discontinuing treatment $[63,64]$. Because pegylated (peg) forms have been shown to have increased tolerance and efficacy in IFN $\alpha$-treated hepatitis patients, there is renewed interest in using pegylated IFN, and clinical trials have been performed to elucidate its role in the upfront and salvage treatment of patients with ET and PV. The objectives included not only high rates of hematologic responses, but also molecular responses in JAK2mutated ET and PV patients. In a phase 2 trial, with a median follow-up of 42 months, a complete hematologic response was achieved in $76 \%$ of patients with PV and $77 \%$ of those with ET. This was accompanied by a complete molecular response (CMR) (i.e., undetectable $J A K 2$ V617F) in $18 \%$ and $17 \%$ of PV and ET patients, respectively [65]. In patients with $\mathrm{PV}$, there was a sustained decrease in the allelic burden, from a median of $64 \%$ to $8 \%$, in those treated for 60 months. In contrast, the majority of ET patients did not experience a significant decrease in the $J A K 2 \mathrm{~V} 617 \mathrm{~F}$ allele burden. In this study, the presence of somatic mutations affected outcomes, with a higher frequency of mutations in genes outside of JAK2, most commonly, TET2, DNMT3A, and ASXL1, in patients failing to achieve a CMR (56\%) versus those achieving CMR (30\%); however, this difference was not statistically significant [65]. Interestingly, in PV, a molecular response does not always accompany a hematologic response
[66]. Recently, responses to IFN have also been reported in patients with $C A L R$-mutated ET [67]. Among $31 C A L R$ mutated ET patients, 77\% achieved a complete hematologic response, and the median CALR burden decreased from $41 \%$ at baseline to $26 \%$ after treatment, with a molecular response rate of $42 \%$ (CMR plus partial MR). In a cohort of CALR-ET patients treated with hydroxyurea or aspirin, the $C A L R$ burden remained stable [67]. Ropeginterferon $\alpha-2 b$ is a monopegylated IFN- $\alpha$ developed for treating PV, which consists of a single positional isomer resulting in an extended elimination half-life, enabling less frequent dosing and improved tolerability, and supporting long-term patient compliance [68]. As reported in data from an open-label, randomized phase study (PROUD-PV and CONTINUATION-PV) that evaluated the efficacy and safety of ropeginterferon $\alpha-2 b$ in comparison to hydroxyurea treatment, ropeginterferon $\alpha$-2b was more effective in achieving durable hematological and molecular remissions than hydroxyurea and was well tolerated during long-term application [69]. In particular, in the CONTINUATION-PV study, patients treated with ropeginterferon $\alpha-2 b$ showed a steady decrease in the mean absolute $J A K 2 \mathrm{~V} 617 \mathrm{~F}$ allele burden to less than half of the baseline level by month 36 . In contrast, in the hydroxyurea group, the reduction was transient and was lost by month 36 , suggesting the possibility of the disease-modifying potential of IFN- $\alpha$-based therapy. Moreover, in a post-hoc analysis, a lower $J A K 2 \mathrm{~V} 617 \mathrm{~F}$ allele burden correlated with a complete hematologic response at 12,24 , and 36 months in the ropeginterferon $\alpha-2 b$ group. However, further study on the clinical relevance of the molecular response during ropeginterferon $\alpha-2 b$ is needed.

\section{NOVEL THERAPIES IN MF}

'Add-on' approaches to ruxolitinib being studied in clinical trials

Unfortunately, since ruxolitinib is insufficient to eliminate the underlying myeloid progenitor clone, 'add-on' approaches to ruxolitinib are being developed for increased efficacy and potential disease-modifying effects (Table 1).

Targeting BET protein: BET proteins regulate key oncogenic pathways, including NF- $\kappa \mathrm{B}$ and TGF- $\beta$ signaling, which are important drivers of pro-inflammatory cytokine expression and bone marrow fibrosis, respectively, and are implicated in MF pathogenesis. Preclinical studies suggest that a combination of CPI-0610, a selective and potent small molecule

Table 1. 'Add-on' approaches to ruxolitinib being studied in clinical trials.

\begin{tabular}{|c|c|c|c|}
\hline Agent (class) & Drug class & Phase (NCT number) & Reference \\
\hline CPI-0610 & BET inhibitor & 2 (NCT02158858) & Mascarenhas et al. [70] \\
\hline Navitoclax & BCL-2/BCL-xL antagonist & 2 (NCT03222609) & Harrison et al. [71] \\
\hline Umbralisib & PI3K $\delta$ inhibitor & 1 (NCT02493530) & Moyo et al. [76] \\
\hline Parsaclisib & PI3K $\delta$ inhibitor & 2 (NCT02718300) & Daver et al. [77] \\
\hline Idelalisib & PI3K $\delta$ inhibitor & 1 (NCT02436135) & - \\
\hline
\end{tabular}


BET inhibitor, together with a JAK inhibitor, can result in the reduction of serum levels of inflammatory cytokines, bone marrow fibrosis (BMF), and mutant cell burden [56]. A phase 2 study of CPI-0610 alone or as an "add-on" to ruxolitinib (CPI-0610+ruxolitinib) (NCT02158858) provided clinical benefits in MF patients with inadequate responses or those refractory to ruxolitinib. Through improvement in BMF and anemia responses, its potential for disease modification has been suggested [70].

Targeting anti-apoptotic protein Bcl-xL: The anti-apoptotic protein $\mathrm{Bcl}-\mathrm{xL}$ is regulated by JAKs and the combined targeting of JAK2. Furthermore, it has been demonstrated that $\mathrm{Bcl} 2 /-\mathrm{xL}$ is synergistic in preclinical JAK2V617F MPN models and helps overcome acquired resistance to ruxolitinib [71]. A phase 2 single-arm, multicenter study (NCT03222609) enrolled adults diagnosed with MF. Eligible patients received $\geq 12$ weeks of continuous ruxolitinib therapy and had persistent splenomegaly that required a new therapy while continuing their current stable dose of ruxolitinib. Navitoclax was initiated at $50 \mathrm{mg} \mathrm{QD}$ and was escalated to $300 \mathrm{mg}$ QD based on tolerability. The primary endpoint was the percentage reduction in the spleen volume (SVR) from baseline. Secondary endpoints included the effect on total symptom score (TSS), BMF, anemia response, and safety. Plasma cytokine levels were measured at baseline and at weeks 12,24 , and 48 . The median percentage change from baseline was calculated for the 140 panel analytes. The combination of navitoclax with ruxolitinib was well tolerated and led to clinically meaningful SVR, improvements in TSS, encouraging reductions in BMF, and cytokine modulation. This study demonstrated that apoptotic induction with navitoclax might be an important therapeutic option for patients with MF to prevent or reverse JAK2 resistance and to modify MF biology [72].

Targeting phosphatidylinositol-3-kinase delta: The phosphatidylinositol-3-kinase/Akt/mammalian target of the rapamycin (PI3K/Akt/mTOR) cascade integrates cellular growth and proliferation signals downstream of JAK-STAT, and constitutive activation of this pathway is central to MPN pathogenesis [73]. Preclinical studies have shown that inhibitors of this pathway, both alone and synergistically in combination with ruxolitinib or fedratinib, reduce proliferation and induce apoptosis of $J A K 2$ V617F/MPLW515L MPN cell lines and primary cells [73-75].

Updated results from a trial in which umbralisib, a selective inhibitor of the delta isoform of PI3K with a superior tolerability profile, was "added on" to ruxolitinib (stable dose for $\geq 8 \mathrm{wk}$ ) in patients with an insufficient response to the latter, were recently presented (NCT02493530) [76]. Two out of 23 patients achieved complete response (CR). An additional 11 patients showed clinical improvement based on anemia, spleen, and/or symptom responses. To note, determination of sub-optimal response to ruxolitinib for patient eligibility for this trial was left up to the physician's discretion. Parsaclisib is another PI3K delta isoform-specific inhibitor that has been studied in combination with ruxolitinib in an ongoing "add on" trial (NCT02718300); how- ever, a sub-optimal response to ruxolitinib is clearly defined in this trial (palpable spleen $>10 \mathrm{~cm}$ or $5-10 \mathrm{~cm}$, with active symptoms of myelofibrosis after at least 6 months of ruxolitinib and with a stable dose over the preceding 8 weeks or longer) [77]. Parsaclisib exhibited a good tolerability profile in this trial, but a switch from daily to weekly dosing after 8 weeks of combination therapy (to mitigate toxicities) appeared to correlate with some loss of response. A similar phase 1 trial of idelalisib added to ruxolitinib (stable dose for $\geq 4 \mathrm{wk}$ ) has been completed (NCT02436135).

\section{Novel agents under study as monotherapies}

Several investigational agents are being studied as monotherapies in ruxolitinib-resistant or ineligible patients.

Telomerase inhibitor: The telomerase inhibitor imetelstat generated considerable excitement when seven durable complete and partial responses from 33 patients with MF, with reversal of $\mathrm{BMF}$ in all four patients who had a complete response, were reported in a pilot study [78]. The results from the phase 2 imetelstat trial in patients with DIPSS intermediate-2/high-risk myelofibrosis who had failed therapy with a JAK inhibitor (NCT02426086) were presented at the 2018 American Society of Hematology (ASH) annual meeting [79]. In this study, two doses of imetelstat $(9.4 \mathrm{mg} / \mathrm{kg}$ or $4.7 \mathrm{mg} / \mathrm{kg} \mathrm{IV}$, every $3 \mathrm{wk}$ ) were administered to 107 patients with intermediate-2 or high-risk MF that was relapsed/refractory to prior JAK inhibitor therapy (i.e., either no reduction in splenomegaly after 12 weeks or worsening splenomegaly at any time after the start of the JAK inhibitor therapy). The lower dose $(4.7 \mathrm{mg} / \mathrm{kg})$ arm $(\mathrm{N}=48)$ was closed to new patient entry due to insufficient activity after an interim analysis, and the patients were allowed a dose escalation. At the time of the clinical cutoff (ASH Annual Meeting 2018), $9.4 \mathrm{mg} / \mathrm{kg}$ administered every 3 weeks resulted in a $10.2 \%$ spleen response and $32 \%$ symptom response. Importantly, after a median follow-up of 22.6 months, the median survival in the $9.4 \mathrm{mg} / \mathrm{kg}$ arm was not reached, while the median OS was 19.9 months in the 4.7 $\mathrm{mg} / \mathrm{kg}$ arm [79]. Although no formal study has reported survival for patients who are truly relapsed/refractory to JAK inhibitors, observed OS after imetelstat therapy was in marked contrast to the 13-14 months reported by several groups studying patients who discontinued ruxolitinib [80-82].

Murine double minute 2 (MDM2) inhibitor: Preclinical studies have shown that JAK2V617F leads to overexpression of murine double minute 2 (MDM2) in MPN [83], and upregulation of MDM2 protects the clonal HSCs driving the disease from apoptosis. An open-label phase 2 trial with the MDM2 inhibitor KRT-232 is currently enrolling patients who failed JAK inhibitor therapy (NCT03662126).

\section{Microenvironment and fibrosis}

Megakaryocytes in PMF exhibit impaired maturation associated with downregulation of the transcription factor GATA1 [84]. These atypical megakaryocytes may contribute to bone marrow fibrosis by releasing cytokines such as trans- 
forming growth factor (TGF)- $\beta$. The aurora kinase A (AURKA) inhibitor alisertib has been shown to promote the differentiation of PMF megakaryocytes and ameliorate bone marrow fibrosis in vivo in mouse models of PMF [85]. In a phase 1 clinical trial of alisertib in 24 patients with myelofibrosis, $63 \%$ of whom had prior exposure to JAK inhibitor therapies, alisertib reduced splenomegaly and symptom burden in $29 \%$ and $32 \%$ of patients, respectively, despite not consistently reducing the degree of inflammatory cytokines. Moreover, correlative studies showed normalization of megakaryocyte morphology, restoration of GATA1 staining, and reduction of BMF in five of seven patients for whom sequential marrows were available. Further study of AURKA inhibition as a therapeutic option for myelofibrosis is planned (NCT02530619) [86].

Fibrosis-driving cells in PMF bone marrow are reported to be clonal, neoplastic, and derived from monocytes [87]. The anti-fibrotic agent, PRM-151, is intravenously administered (every $4 \mathrm{wk}$ ) and recombinant pentraxin-2 molecule, also known as serum amyloid protein. The results from 18 patients, 9 of whom received PRM-151 alone and 9 that received a combination with ruxolitinib in an open-label extension study, are presented. The median time of study was 30.9 months, and the drug was well tolerated. The mean best percent change (by palpation) in spleen size from baseline was $-37 \%$, with a median percent reduction of $-26.1 \%$. The mean best percent improvement in the MPN-SAF TSS was $-54 \%$, with a median percent reduction of TSS of $-64 \%$. Interestingly, even the patients on PRM-151 monotherapy showed similar benefits in terms of spleen size and symptom burden as those receiving PRM-151+ruxolitinib. In addition, an overall improvement in the BM reticulin and collagen fibrosis grade was observed [88].

There are some potential strategies aimed at reversing bone marrow fibrosis. Galunisertib, a small-molecule inhibitor of the TGF- $\beta$ receptor 1 kinase ALK5, blocks excessive collagen production in $J A K 2 \mathrm{~V} 617 \mathrm{~F}$ and $M P L \mathrm{~W} 515 \mathrm{~L}$ mouse models [89]. Sotatercept and luspatercept are activin receptor type IIA ligand traps designed to sequester natural ligands to the TGF- $\beta$ receptor and inhibit signaling. These agents are currently in clinical trials for patients with $\mathrm{MF}$ and anemia $[90,91]$.

\section{Immunotherapy}

The effect of recombinant interferon to prevent the development of marked splenomegaly, anemia, and florid myelofibrosis in early myelofibrosis was tested [92]. Early data on the combination of ruxolitinib and pegylated IFN- $\alpha$ from the ongoing RUXOPEG study showed that this combination was generally well tolerated, and the preliminary efficacy results were encouraging [93].

Mutant calreticulin binds to the thrombopoietin receptor, MPL (requirement for the lectin-dependent function of mutant calreticulin to bind to the extracellular domain of MPL) to serve as a potential tumor antigen in MPN [94]. This led to the development of novel, vaccine-based approaches to target this immunogenic mutant protein $[95,96]$, but these have not yet entered clinical practice.

\section{CONCLUSIONS}

Although the identification of driver mutations in $J A K 2$, $C A L R$, and $M P L$ of classical MPN have contributed to a better understanding of classical MPN pathogenesis, no drug therapy has clearly been proven to be disease-modifying. Over the past decade, it has been shown that treatment with ruxolitinib improves splenomegaly and associated symptoms regardless of driver mutation status and survival advantage in patients with intermediate-2/high-risk MF. However, drug-related cytopenias, refractory, resistance to ruxolitinib after 2-3 years of therapy, and disease progression remain a challenge $[80,97]$. In particular, patients have shown poor survival after ruxolitinib discontinuation, particularly in the presence of clonal evolution and/or declining platelet counts, while on ruxolitinib [80-82, 98]. Therefore, clinical studies to identify novel agents with disease-modifying properties are underway as either solo or 'add-on' therapies to ruxolitinib. In addition, strategies targeting bone marrow fibrosis and immunotherapeutic approaches are also being studied.

\section{Authors' Disclosures of Potential Conflicts of Interest}

No potential conflicts of interest relevant to this article were reported.

\section{REFERENCES}

1. Nangalia J, Green AR. Myeloproliferative neoplasms: from origins to outcomes. Blood 2017;130:2475-83.

2. Vainchenker W, Kralovics R. Genetic basis and molecular pathophysiology of classical myeloproliferative neoplasms. Blood 2017;129:667-79.

3. Grinfeld J, Nangalia J, Green AR. Molecular determinants of pathogenesis and clinical phenotype in myeloproliferative neoplasms. Haematologica 2017;102:7-17.

4. Verstovsek S, Mesa RA, Gotlib J, et al. A double-blind, placebocontrolled trial of ruxolitinib for myelofibrosis. N Engl J Med 2012;366:799-807.

5. Harrison C, Kiladjian JJ, Al-Ali HK, et al. JAK inhibition with ruxolitinib versus best available therapy for myelofibrosis. N Engl J Med 2012;366:787-98.

6. Vannucchi AM, Kiladjian JJ, Griesshammer M, et al. Ruxolitinib versus standard therapy for the treatment of polycythemia vera. N Engl J Med 2015;372:426-35.

7. Cancer Genome Atlas Research Network, Ley TJ, Miller C, et al. Genomic and epigenomic landscapes of adult de novo acute myeloid leukemia. N Engl J Med 2013;368:2059-74.

8. Papaemmanuil E, Gerstung M, Bullinger L, et al. Genomic classification and prognosis in acute myeloid leukemia. $\mathrm{N}$ Engl J Med 2016;374:2209-21. 
9. Papaemmanuil E, Gerstung M, Malcovati L, et al. Clinical and biological implications of driver mutations in myelodysplastic syndromes. Blood 2013;122:3616-27; quiz 3699.

10. Bejar R, Stevenson KE, Caughey B, et al. Somatic mutations predict poor outcome in patients with myelodysplastic syndrome after hematopoietic stem-cell transplantation. J Clin Oncol 2014;32: 2691-8.

11. Jaiswal S, Fontanillas P, Flannick J, et al. Age-related clonal hematopoiesis associated with adverse outcomes. N Engl J Med 2014;371:2488-98.

12. McKerrell T, Park N, Moreno T, et al. Leukemia-associated somatic mutations drive distinct patterns of age-related clonal hemopoiesis. Cell Rep 2015;10:1239-45.

13. Xie M, Lu C, Wang J, et al. Age-related mutations associated with clonal hematopoietic expansion and malignancies. Nat Med 2014;20:1472-8.

14. Jang MA, Choi CW. Recent insights regarding the molecular basis of myeloproliferative neoplasms. Korean J Intern Med 2020; 35:1-11.

15. Rumi E, Cazzola M. How I treat essential thrombocythemia. Blood 2016;128:2403-14.

16. Spivak JL. How I treat polycythemia vera. Blood 2019;134:341-52.

17. Shammo JM, Stein BL. Mutations in MPNs: prognostic implications, window to biology, and impact on treatment decisions. Hematology Am Soc Hematol Educ Program 2016; 2016:552-60.

18. Verstovsek S, Kantarjian H, Mesa RA, et al. Safety and efficacy of INCB018424, a JAK1 and JAK2 inhibitor, in myelofibrosis. N Engl J Med 2010;363:1117-27.

19. Tefferi A. Primary myelofibrosis: 2019 update on diagnosis, riskstratification and management. Am J Hematol 2018;93:1551-60.

20. Devlin R, Gupta V. Myelofibrosis: to transplant or not to transplant? Hematology Am Soc Hematol Educ Program 2016; 2016:543-51.

21. James C, Ugo V, Le Couédic JP, et al. A unique clonal JAK2 mutation leading to constitutive signalling causes polycythaemia vera. Nature 2005;434:1144-8.

22. Baxter EJ, Scott LM, Campbell PJ, et al. Acquired mutation of the tyrosine kinase JAK2 in human myeloproliferative disorders. Lancet 2005;365:1054-61.

23. Kralovics R, Passamonti F, Buser AS, et al. A gain-of-function mutation of JAK2 in myeloproliferative disorders. N Engl J Med 2005;352:1779-90.

24. Levine RL, Wadleigh M, Cools J, et al. Activating mutation in the tyrosine kinase JAK2 in polycythemia vera, essential thrombocythemia, and myeloid metaplasia with myelofibrosis. Cancer Cell 2005; 387-97.

25. Pardanani AD, Levine RL, Lasho T, et al. MPL515 mutations in myeloproliferative and other myeloid disorders: a study of 1182 patients. Blood 2006;108:3472-6.

26. Klampfl T, Gisslinger H, Harutyunyan AS, et al. Somatic mutations of calreticulin in myeloproliferative neoplasms. N Engl J Med 2013;369:2379-90.

27. Nangalia J, Massie CE, Baxter EJ, et al. Somatic CALR mutations in myeloproliferative neoplasms with nonmutated JAK2. N Engl J Med 2013;369:2391-405.

28. Scott LM, Tong W, Levine RL, et al. JAK2 exon 12 mutations in polycythemia vera and idiopathic erythrocytosis. N Engl J Med 2007;356:459-68.

29. Staerk J, Lacout C, Sato T, Smith SO, Vainchenker W, Constantinescu SN. An amphipathic motif at the transmembrane-cytoplasmic junction prevents autonomous activation of the thrombopoietin receptor. Blood 2006;107:1864-71.

30. Pikman Y, Lee BH, Mercher T, et al. MPLW515L is a novel somatic activating mutation in myelofibrosis with myeloid metaplasia. PLoS Med 2006;3:e270.

31. Defour JP, Chachoua I, Pecquet C, Constantinescu SN. Oncogenic activation of MPL/thrombopoietin receptor by 17 mutations at W515: implications for myeloproliferative neoplasms. Leukemia 2016;30:1214-6.

32. Rumi E, Pietra D, Ferretti V, et al. JAK2 or CALR mutation status defines subtypes of essential thrombocythemia with substantially different clinical course and outcomes. Blood 2014;123:1544-51.

33. Rumi E, Pietra D, Pascutto C, et al. Clinical effect of driver mutations of JAK2, CALR, or MPL in primary myelofibrosis. Blood 2014;124:1062-9.

34. Cabagnols X, Defour JP, Ugo V, et al. Differential association of calreticulin type 1 and type 2 mutations with myelofibrosis and essential thrombocytemia: relevance for disease evolution. Leukemia 2015;29:249-52.

35. Tefferi A, Lasho TL, Abdel-Wahab O, et al. IDH1 and IDH2 mutation studies in 1473 patients with chronic-, fibrotic- or blast-phase essential thrombocythemia, polycythemia vera or myelofibrosis. Leukemia 2010;24:1302-9.

36. Abdel-Wahab O, Mullally A, Hedvat C, et al. Genetic characterization of TET1, TET2, and TET3 alterations in myeloid malignancies. Blood 2009;114:144-7.

37. Ernst T, Chase AJ, Score J, et al. Inactivating mutations of the histone methyltransferase gene EZH2 in myeloid disorders. Nat Genet 2010;42:722-6.

38. Carbuccia N, Murati A, Trouplin V, et al. Mutations of ASXL1 gene in myeloproliferative neoplasms. Leukemia 2009;23:2183-6.

39. Stein BL, Williams DM, O'Keefe C, et al. Disruption of the ASXL1 gene is frequent in primary, post-essential thrombocytosis and post-polycythemia vera myelofibrosis, but not essential thrombocytosis or polycythemia vera: analysis of molecular genetics and clinical phenotypes. Haematologica 2011;96:1462-9.

40. Lasho TL, Finke CM, Hanson CA, et al. SF3B1 mutations in primary myelofibrosis: clinical, histopathology and genetic correlates among 155 patients. Leukemia 2012;26:1135-7.

41. Lasho TL, Jimma T, Finke CM, et al. SRSF2 mutations in primary myelofibrosis: significant clustering with IDH mutations and independent association with inferior overall and leukemia-free survival. Blood 2012;120:4168-71.

42. Stegelmann F, Bullinger L, Schlenk RF, et al. DNMT3A mutations in myeloproliferative neoplasms. Leukemia 2011;25:1217-9.

43. Yoshida K, Sanada M, Shiraishi Y, et al. Frequent pathway mutations of splicing machinery in myelodysplasia. Nature 2011;478:64-9.

44. Harutyunyan A, Klampfl T, Cazzola M, Kralovics R. p53 lesions in leukemic transformation. N Engl J Med 2011;364:488-90.

45. Klampfl T, Harutyunyan A, Berg T, et al. Genome integrity of myeloproliferative neoplasms in chronic phase and during disease progression. Blood 2011;118:167-76. 
46. Tefferi A, Lasho TL, Guglielmelli $\mathrm{P}$, et al. Targeted deep sequencing in polycythemia vera and essential thrombocythemia. Blood Adv 2016;1:21-30.

47. Tefferi A, Guglielmelli P, Lasho TL, et al. Mutation-enhanced international prognostic systems for essential thrombocythaemia and polycythaemia vera. Br J Haematol 2020;189:291-302.

48. Guglielmelli P, Lasho TL, Rotunno G, et al. The number of prognostically detrimental mutations and prognosis in primary myelofibrosis: an international study of 797 patients. Leukemia 2014;28:1804-10.

49. Delhommeau F, Dupont S, Della Valle V, et al. Mutation in TET2 in myeloid cancers. N Engl J Med 2009;360:2289-301.

50. Ortmann CA, Kent DG, Nangalia J, et al. Effect of mutation order on myeloproliferative neoplasms. N Engl J Med 2015;372:601-12.

51. Figueroa ME, Abdel-Wahab O, Lu C, et al. Leukemic IDH1 and IDH2 mutations result in a hypermethylation phenotype, disrupt TET2 function, and impair hematopoietic differentiation. Cancer Cell 2010;18:553-67.

52. Kamminga LM, Bystrykh LV, de Boer A, et al. The Polycomb group gene Ezh2 prevents hematopoietic stem cell exhaustion. Blood 2006;107:2170-9.

53. Abdel-Wahab O, Adli M, LaFave LM, et al. ASXL1 mutations promote myeloid transformation through loss of PRC2-mediated gene repression. Cancer Cell 2012;22:180-93.

54. Schaub FX, Looser R, Li S, et al. Clonal analysis of TET2 and JAK2 mutations suggests that TET2 can be a late event in the progression of myeloproliferative neoplasms. Blood 2010;115:2003-7.

55. Sashida G, Wang C, Tomioka T, et al. The loss of Ezh2 drives the pathogenesis of myelofibrosis and sensitizes tumor-initiating cells to bromodomain inhibition. J Exp Med 2016;213:1459-77.

56. Kleppe M, Koche R, Zou L, et al. Dual targeting of oncogenic activation and inflammatory signaling increases therapeutic efficacy in myeloproliferative neoplasms. Cancer Cell 2018;33: 29-43, e7.

57. Brecqueville M, Rey J, Bertucci F, et al. Mutation analysis of ASXL1, CBL, DNMT3A, IDH1, IDH2, JAK2, MPL, NF1, SF3B1, SUZ12, and TET2 in myeloproliferative neoplasms. Genes Chromosomes Cancer 2012;51:743-55.

58. Kim E, Ilagan JO, Liang Y, et al. SRSF2 mutations contribute to myelodysplasia by mutant-specific effects on exon recognition. Cancer Cell 2015;27:617-30.

59. Khan SN, Jankowska AM, Mahfouz R, et al. Multiple mechanisms deregulate EZH2 and histone H3 lysine 27 epigenetic changes in myeloid malignancies. Leukemia 2013;27:1301-9.

60. Rampal R, Ahn J, Abdel-Wahab O, et al. Genomic and functional analysis of leukemic transformation of myeloproliferative neoplasms. Proc Natl Acad Sci U S A 2014;111:E5401-10.

61. Sood R, Kamikubo Y, Liu P. Role of RUNX1 in hematological malignancies. Blood 2017;129:2070-82.

62. Beer PA, Delhommeau F, LeCouedic JP, et al. Two routes to leukemic transformation after a JAK2 mutation-positive myeloproliferative neoplasm. Blood 2010;115:2891-900.

63. Gisslinger $\mathrm{H}$, Ludwig $\mathrm{H}$, Linkesch $\mathrm{W}$, Chott A, Fritz E, Radaszkiewicz T. Long-term interferon therapy for thrombocytosis in myeloproliferative diseases. Lancet 1989;1:634-7.

64. Silver RT. Long-term effects of the treatment of polycythemia vera with recombinant interferon-alpha. Cancer 2006;107:451-8.
65. Quintás-Cardama A, Abdel-Wahab O, Manshouri T, et al. Molecular analysis of patients with polycythemia vera or essential thrombocythemia receiving pegylated interferon alpha-2a. Blood 2013;122:893-901.

66. Kuriakose E, Vandris K, Wang YL, et al. Decrease in JAK2 V617F allele burden is not a prerequisite to clinical response in patients with polycythemia vera. Haematologica 2012;97:538-42.

67. Verger E, Cassinat B, Chauveau A, et al. Clinical and molecular response to interferon-alpha therapy in essential thrombocythemia patients with CALR mutations. Blood 2015;126:2585-91.

68. Gisslinger H, Zagrijtschuk O, Buxhofer-Ausch V, et al. Ropeginterferon alfa- $2 b$, a novel IFNalpha- $2 b$, induces high response rates with low toxicity in patients with polycythemia vera. Blood 2015;126:1762-9.

69. Gisslinger H, Klade C, Georgiev P, et al. Ropeginterferon alfa-2b versus standard therapy for polycythaemia vera (PROUD-PV and CONTINUATION-PV): a randomised, non-inferiority, phase 3 trial and its extension study. Lancet Haematol 2020;7:e196-208.

70. Mascarenhas J, Kremyanskaya M, Hoffman R, et al. MANIFEST, a phase 2 study of CPI-0610, a bromodomain and extraterminal domain inhibitor (BETi), as monotherapy or "Add-on" to ruxolitinib, in patients with refractory or intolerant advanced myelofibrosis. Blood (ASH Annual Meeting Abstracts) 2019; 134(Suppl):670.

71. Waibel M, Solomon VS, Knight DA, et al. Combined targeting of JAK2 and Bcl-2/Bcl-xL to cure mutant JAK2-driven malignancies and overcome acquired resistance to JAK2 inhibitors. Cell Rep 2013;5:1047-59.

72. Harrison C, Garcia JS, Mesa R, et al. Navitoclax in combination with ruxolitinib in patients with primary or secondary myelofibrosis; a phase 2 study. 25th EHA Congress Abstracts 2020:EP1081.

73. Khan I, Huang Z, Wen Q et al. AKT is a therapeutic target in myeloproliferative neoplasms. Leukemia 2013;27:1882-90.

74. Bogani C, Bartalucci N, Martinelli S, et al. mTOR inhibitors alone and in combination with JAK2 inhibitors effectively inhibit cells of myeloproliferative neoplasms. PLoS One 2013;8:e54826.

75. Fiskus W, Verstovsek S, Manshouri T, et al. Dual PI3K/ AKT/mTOR inhibitor BEZ235 synergistically enhances the activity of JAK2 inhibitor against cultured and primary human myeloproliferative neoplasm cells. Mol Cancer Ther 2013;12: 577-88.

76. Moyo T, Palmer J, Huang Y, et al. Resurrecting response to ruxolitinib: a phase I study testing the combination of ruxolitinib and the PI3K delta inhibitor umbralisib in ruxolitinibexperienced myelofibrosis. 23rd EHA Congress Abstracts 2018:S133.

77. Daver NG, Kremyanskaya M, O'Connell C, et al. A phase 2 study of the safety and efficacy of INCB050465, a selective PI3K $\delta$ inhibitor, in combination with ruxolitinib in patients with myelofibrosis. Blood (ASH Annual Meeting Abstracts) 2018; 132(Suppl):353.

78. Tefferi A, Lasho TL, Begna KH, et al. A pilot study of the telomerase inhibitor imetelstat for myelofibrosis. N Engl J Med 2015; 373:908-19.

79. Mascarenhas J, Komrokji RS, Cavo M, et al. Imetelstat is effective treatment for patients with intermediate-2 or high-risk 
myelofibrosis who have relapsed on or are refractory to Janus Kinase inhibitor therapy: results of a phase 2 randomized study of two dose levels. Blood (ASH Annual Meeting Abstracts) 2018; 132(Suppl):685.

80. Kuykendall AT, Shah S, Talati C, et al. Between a rux and a hard place: evaluating salvage treatment and outcomes in myelofibrosis after ruxolitinib discontinuation. Ann Hematol 2018;97:435-41.

81. Palandri F, Breccia M, Bonifacio M, et al. Life after ruxolitinib: reasons for discontinuation, impact of disease phase, and outcomes in 218 patients with myelofibrosis. Cancer 2020; 126:1243-52.

82. Newberry KJ, Patel K, Masarova L, et al. Clonal evolution and outcomes in myelofibrosis after ruxolitinib discontinuation. Blood 2017;130:1125-31.

83. Nakatake M, Monte-Mor B, Debili N, et al. JAK2(V617F) negatively regulates $\mathrm{p} 53$ stabilization by enhancing MDM2 via La expression in myeloproliferative neoplasms. Oncogene 2012;31: 1323-33.

84. Gilles L, Arslan AD, Marinaccio C, et al. Downregulation of GATA1 drives impaired hematopoiesis in primary myelofibrosis. J Clin Invest 2017;127:1316-20.

85. Wen QJ, Yang Q Goldenson B, et al. Targeting megakaryocyticinduced fibrosis in myeloproliferative neoplasms by AURKA inhibition. Nat Med 2015;21:1473-80.

86. Gangat N, Marinaccio C, Swords R, et al. Aurora kinase a inhibition provides clinical benefit, normalizes megakaryocytes, and reduces bone marrow fibrosis in patients with myelofibrosis: a phase I trial. Clin Cancer Res 2019;25:4898-906.

87. Verstovsek S, Manshouri T, Pilling D, et al. Role of neoplastic monocyte-derived fibrocytes in primary myelofibrosis. J Exp Med 2016;213:1723-40.

88. Verstovsek S, Hasserjian RP, Pozdnyakova O, et al. PRM-151 in myelofibrosis: efficacy and safety in an open label extension study. Blood (ASH Annual Meeting Abstracts) 2018;132(Suppl):686.

89. Yue L, Bartenstein M, Zhao W, et al. Efficacy of ALK5 inhibition in myelofibrosis. JCI Insight 2017;2:e90932.
90. Bose P, Daver N, Jabbour EJ, et al. Phase-2 study of sotatercept (ACE-011) in myeloproliferative neoplasm-associated myelofibrosis and anemia. Blood (ASH Annual Meeting Abstracts) 2016; 128(Suppl):478.

91. Mesa RA, Barosi G, Harrison CN, et al. A phase 2, multicenter, open-label study of the safety and efficacy of luspatercept in subjects with myeloproliferative neoplasm (MPN)-associated myelofibrosis and anemia with or without RBC transfusion dependence. J Clin Oncol (ASCO Annual Meeting Abstracts) 2018;36(Suppl):TPS7083.

92. How J, Hobbs G. Use of interferon alfa in the treatment of myeloproliferative neoplasms: perspectives and review of the literature. Cancers (Basel) 2020;12:1954.

93. Kiladjian JJ, Soret-Dulphy J, Resche-Rigon M, et al. Ruxopeg, a multi-center bayesian phase $1 / 2$ adaptive randomized trial of the combination of ruxolitinib and pegylated interferon alpha 2a in patients with myeloproliferative neoplasm (MPN)-associated myelofibrosis. Blood (ASH Annual Meeting Abstracts) 2018; 132(Suppl):581.

94. Elf S, Abdelfattah NS, Baral AJ, et al. Defining the requirements for the pathogenic interaction between mutant calreticulin and MPL in MPN. Blood 2018;131:782-6.

95. Holmström MO, Riley CH, Svane IM, Hasselbalch HC, Andersen MH. The CALR exon 9 mutations are shared neoantigens in patients with CALR mutant chronic myeloproliferative neoplasms. Leukemia 2016;30:2413-6.

96. Holmström MO, Martinenaite E, Ahmad SM, et al. The calreticulin (CALR) exon 9 mutations are promising targets for cancer immune therapy. Leukemia 2018;32:429-37.

97. Verstovsek S, Mesa RA, Gotlib J, et al. Long-term treatment with ruxolitinib for patients with myelofibrosis: 5 -year update from the randomized, double-blind, placebo-controlled, phase 3 COMFORTI trial. J Hematol Oncol 2017;10:55.

98. Lundberg P, Karow A, Nienhold R, et al. Clonal evolution and clinical correlates of somatic mutations in myeloproliferative neoplasms. Blood 2014;123:2220-8. 